\title{
La Carrera Profesional desde el punto de vista de un Médico Clínico
}

a Médico de Canencia de la Sierra, Garganta de los Montes y El Cuadrón (Madrid), Equipo CESCA, Madrid

Correspondencia: Juan Gérvas Camacho, e-mail: jgervasc@meditex.es

Recibido el 8 de marzo de 2008.

Aceptado para su publicación el 14 de abril de 2008.

\author{
Juan Gérvas Camacho ${ }^{a}$
}

\begin{abstract}
RESUMEN
La carrera profesional ha sido largamente esperada como un incentivo para ampliar el horizonte profesional y para mejorar la calidad de los servicios prestados a los pacientes (y por consecuencia, para mejorar la salud de la población). Pero no parece que la carrera profesional en la práctica sea la carrera profesional en la teoría, ni que los hechos hayan respondido a las expectativas. La carrera profesional ha sido una oportunidad perdida, pues no va ligada a cambios en el trabajo, ha incrementado las diferencias autonómicas, ha hecho más iguales a los desiguales, ha discriminado a los interinos, y no tendrá impacto en la salud de la población.

Palabras clave: Incentivos. Desarrollo Profesional. Carrera profesional. Formación continuada. Atención Primaria.
\end{abstract}

\section{ABSTRACT}

A career in medicine from a clinical physician's perspective

For a long time, a career in medicine has been considered as an incentive to broaden professional horizons and to improve the quality of services that patients receive (and consequently, to improve the health of the population). However, the reality of a professional career in medicine does not completely comply with the theory, and does not satisfy expectations. A career in medicine is a lost opportunity since it is not related to job changes, the differences between the autonomous regions have become greater, individual differences are being ignored, there is discrimination against physicians working for the National Health Service and the career does not have a positive impact on population health.

Key words. Incentives. Professional develgsment. Professional progression. Continuous medical education. Primary Care.

\section{INTRODUCCIÓN}

La carrera profesional ha sido largamente esperada como un incentivo para ampliar el horizonte profesional y para mejorar la calidad de los servicios prestados a los pacientes (y por consecuencia, para mejorar la salud de la población) ${ }^{1-3}$. Pero no parece que la carrera profesional en la práctica sea la carrera profesional en la teoría, ni que los hechos hayan respondido a las expectativas. Para valorar la carrera conviene contestar a cuatro preguntas, al menos:

1. ¿Ha cambiado tu trabajo en algo? ¿Has "ascendido"? ¿Has conseguido una determinada situación con la carrera profesional? En mi caso, he conseguido el máximo grado de la carrera profesional. Pero nada ha cambiado en mi trabajo diario. Sigo haciendo exactamente lo mismo. A final de mes me han subido el sueldo. Una subida menor, evidentemente, pues se está introduciendo a pequeñas "dosis". Eso parece ser todo. Tampoco esperaba mucho, pues sólo se valoró mi antigüedad en el puesto de trabajo, y con treinta y tres años de servicios prestados me "merecía" el máximo. Ya sé que la experiencia es un grado, pero parece que nada más. Para mí, pues, la carrera profesional ha sido como una especie de "trienio", un extra a este respecto. No está mal. ¿O sí está mal?

2. Puesto que la carrera profesional sólo ha significado hasta ahora antigüedad y dinero, ¿has comprobado qué representa en tu caso en dinero la carrera profesional? ¿Has comparado tu caso con el de compañeros de otras CCAA? Hay tales diferencias que, aunque parezca mentira, la carrera profesional puede aumentar las dificultades para la libre circulación de los médicos. Las transferencias tienen ventajas e inconvenientes, pero no parecen estar resolviendo problemas básicos, como las desigualdades en salud y el re-equilibrio de la oferta de médicos ${ }^{4-5}$. La carrera profesional se ha convertido en un nuevo obstáculo, por su disparidad entre $\mathrm{CCAA}^{6}$. ¿Estamos de nuevo en los reinos de taifas, pero sin el árabe como lengua común? 
3. ¿Qué esperabas de la carrera profesional? ¿Lo ha cumplido en algún aspecto? Desde luego, para mí la carrera profesional ha sido un chasco, una desilusión más. Nos ha igualado a todos, y ha discriminado injustamente, algo difícil de lograr al tiempo. Nos ha igualado por lo fácil, por la antigüedad. $Y$ ha discriminado a todos lo que no tienen una plaza fija. Nos han hecho más burócratas, más pendientes del simple paso del tiempo. Hay quien habla de "trienios chusqueros", de los que se consiguen sólo con "estar". Ni se ha tenido en cuenta la calidad del trabajo, ni se ha valorado mérito alguno. ¿Esperábamos demasiado, o nos han ofrecido poco?

4. Nuestro trabajo es importante desde el punto de vista social, y, puesto que somos parte del Estado de Bienestar, como médicos en un sistema sanitario público, ¿qué supone para la sociedad la carrera profesional? En mi opinión y en mi práctica, un aumento del gasto sin impacto en la salud de la población. Si trabajo como trabajaba, si la carrera profesional no ha tenido impacto alguno en $\mathrm{mi}$ actividad laboral, hay que esperar que nada cambie, tampoco, en la sociedad para la que trabajo. ¿Es posible que sólo sea una especie de aumento vergonzoso y encubierto del sueldo?

En este texto intentaré dar respuesta a las cuatro cuestiones planteadas, por orden inverso al de su enunciado.

\section{LAS INSTITUCIONES COMO INCENTIVOS}

En las teorías económicas y sociológicas las instituciones son las reglas de juego que gobiernan la sociedad y sus organizaciones. Unas reglas transparentes y lógicas favorecen el buen trabajo, y la adhesión del profesional a los objetivos de la organización.

En las organizaciones sanitarias, además de las reglas generales caben algunas particulares, que se refieren a las normas clínicas, al impacto del Estado de Bienestar, y a las relaciones de los profesionales con las industrias (farmacéuticas, de servicios y tecnológicas) ${ }^{7}$. Estas reglas particulares no excluyen las generales, del estilo de las que se refieren a la carrera profesional.

Hay que ver, pues, la carrera profesional en el contexto de las reglas de juego que permiten a las organizaciones la consecución de aquellos bienes que más valora la sociedad (y a un precio que la misma esté dispuesta a aceptar). En este sentido, la carrera profesional, al no cambiar las reglas de juego, no tiene impacto esperable en la consecución de los objetivos de la organización sanitaria. Se pierde con ello un poderosísimo incentivo.

Todos nos adaptamos al ambiente. Es una norma esencial para supervivir. Por ello, si el ambiente en una organización es de mediocridad, la media del trabajo será mediocre. Y, por supuesto, si el ambiente es excepcionalmente positivo, todos daremos lo mejor de nosotros mismos. La historia de los incentivos con los que se busca científicamente la mejora ambiental es apenas centenaria ${ }^{8}$. Pero el lograr un ambiente positivo ha sido desde siempre un objetivo de cualquier organización, se llame tribu, familia, expedición, ciudad, ejército o universidad.

El ambiente se crea con las reglas de juego, y con personas que las cumplen y las mejoran. Por ello son clave las instituciones democráticas, las reglas de juego que implican una ética. La democracia no es el cumplimiento de rituales (elecciones, parlamentos y demás) sino formas negociadas de resolución de conflictos en las que se transfieren recursos según capacidad y necesidad, con respeto a las minorías ${ }^{9,10}$. La democracia sólo existe cuando hay: 1) libertades políticas (prensa, reunión y demás), 2) oportunidades sociales (educación, servicios sanitarios), 3) garantías de transparencia (contra la corrupción), 4) facilidades económicas (comercio) y 5) seguridad protectora (paro, pensiones) ${ }^{9}$. En este sentido, la democracia ayuda a promover salud, a incrementar la salud de las poblaciones, incluso si se corrige por riqueza y educación ${ }^{11}$. La democracia logra su efecto sobre la salud a través de unas reglas de juego transparentes y lógicas. La falta de lógica de la carrera profesional en las organizaciones sanitarias públicas hace un flaco favor al conjunto de la sociedad.

\section{SOMOS IGUALES, PERO NO DEMASIADO IGUALES, NI DEMASIADO DESIGUALES}

La igualdad no es tratar a todos por igual. Para demostrarlo sirva el clásico ejemplo de dar el mismo número de zapatos a todos los españoles, con el objetivo de igualarlos ante la necesidad de calzarlos. En el campo sanitario es exactamente lo mismo. No tiene sentido igualar a todo el mundo a través, por ejemplo, del transplante de riñón. La sociedad admite y promueve el establecimiento de unos criterios "justos", como el que prefiere ante el transplante a aquel paciente que más se pueda beneficiar (co-morbilidad, evolución de la enfermedad de base y demás). La verdadera igualdad se logra cuando todos tenemos realmente las mismas posibilidades de desarrollarnos como personas $\mathrm{y} / \mathrm{o}$ profesionales, un ideal bien lejano, pero que marca el rumbo en todas las sociedades democráticas.

La carrera profesional al tiempo nos ha igualado y discriminado en exceso. No es fácil lograrlo, pero se ha conseguido. Ha igualado a todos, según antigüedad, y ha discriminado a los interinos, por no tener plaza. Prometen arreglarlo en el futuro, pues se ve la carrera profesional como un desarrollo que se aplica de una vez a los que ya trabajan con plaza fija ("titulares", o "con plaza en propiedad"), y en el futuro se irá aplicando con otros criterios, más racionales, a los que se vayan incorporando. Pero, ¿por qué esperar al futuro, cuando ya les afecta de pleno a muchos médicos que tienen incluso 30 años, y a otros que están en los 60 ? Para aquéllos hay demasiado futuro; para estos, poca esperanza. Además, el "efecto senda" (el camino ya establecido) tendrá un peso que hace dudar de las buenas intenciones. 
La carrera profesional era esperada como mecanismo para reconocer la desigualdad, pues no hay nada más alienante que tratar como iguales a los desiguales. $Y$ desiguales somos todos. No queremos ser tratados como iguales, sino que a todos se nos ofrezcan posibilidades de desarrollo acordes con nuestras capacidades.

Es demagógico el ofrecer a todos lo mismo, el igualar y no discriminar, el emplear un aparente principio justo igualatorio. Si empleamos para medir la igualdad cuatro criterios, como el trabajo clínico, la docencia, la investigación y la microgestión, es evidente la desigualdad entre médicos.

Por supuesto, creo que lo básico al comparar médicos clínicos es su trabajo diario como clínicos, y que las otras tres "patas" del trabajo deberían ponderar mucho menos. Personalmente esperaba de la carrera profesional no sólo un reconocimiento, una "escala", sino un horizonte profesional, un incentivo a la calidad del trabajo clínico, y una motivación para la actividad diaria $^{1-3}$. Nada de todo ello ha llegado.

Ni siquiera nos prometen medios adecuados para valorar la actividad clínica diaria. Se piensa, como mucho, en la re-certificación, pero hay muchas otras formas y maneras de valorar el compromiso del médico con sus pacientes. Por ejemplo, ¿se ha considerado en primaria algo tan básico como la promoción y el mantenimiento de la longitudinalidad? Un médico general/de familia ofrece longitudinalidad cuando permanece en el mismo puesto de trabajo (pueblo, centro de salud) a lo largo de décadas, y cuando ofrece una polivalencia que permita dar respuesta a la mayor parte de los problemas de salud del paciente y de su familia (el médico general/de familia es generalista en la práctica, atiende múltiples problemas en cada encuentro, y hace desde seguimiento del embarazo a cirugía menor, desde terapia de familia a atención de terminales a domicilio). Se puede incentivar la longitudinalidad, pero hace falta tenerlo en cuenta ${ }^{12}$. En la carrera profesional este aspecto básico se ignora, como otros muchos que podrían servir de indicador del desempeño clínico. Por supuesto, es más fácil "medir" publicaciones, horas de docencia, tutorización de alumnos y residentes y demás actividades no clínicas, pero el médico de a pie debe ser valorado, fundamentalmente, por su trabajo clínico.

Los médicos interinos son cuenta aparte. Muchos pueden trabajar durante años codo a codo con los médicos propietarios, pero las reglas del juego no son las mismas para ambos grupos. Nada más violento que reservar la carrera profesional para los fijos, y dejar en el limbo a los interinos. Si todos trabajamos para la misma organización, conviene no discriminar más de lo necesario a los que tienen diferente situación laboral. Los propietarios disfrutan de ventajas múltiples, especialmente de la seguridad de tener una plaza fija. Al rechazar la incorporación de los interinos a la carrera profesional los alejamos de los objetivos de la organización sanitaria, al tiempo de aplicarles reglas del juego que no honran al que trabaja como debe.

\section{COMUNIDADES, ¿AUTÓNOMAS O ANTÓNIMAS?}

En España las CCAA han recibido las transferencias sanitarias y de facto se están construyendo 18 sistemas sanitarios, en los que ni siquiera se busca la compatibilidad de las historias clínicas electrónicas. La descentralización tiene ventajas, pero se está haciendo poco para compensar las desventajas ${ }^{4,5}$. Además, las opciones políticas que gobiernan las CCAA reflejan en exceso sus preferencias en el sector sanitario, lo que conlleva grandes diferencias especialmente en lo que respecta a la atención primaria ${ }^{13-15}$.

Con estos mimbres, el cesto de la carrera profesional tenía que ser muy dispar, según Comunidad Autónoma. Y así es ${ }^{6}$. Por supuesto, la homologación tendrá que ser automática cuando un médico cambie de Comunidad Autónoma, aunque no están claros los procedimientos. Pero aunque la homologación sea imperativa, las diferencias en origen pueden llegar a ser insalvables, pues ni siquiera existe acuerdo respecto a las definiciones. Además, los méritos se miden y seleccionan de diferente manera, con distinto énfasis en la autoevaluación. También es diferente el tiempo mínimo en cada categoría. Y diferente la existencia de pruebas de competencia para el cambio de niveles. Sin hablar de que en algunas CCAA, pero no en otras, existe la reversibilidad de la situación en una categoría o nivel (se puede perder en determinadas circunstancias, y volver al previo). Y hay más diferencias, cuyo análisis exigiría un tratado; por ejemplo, en Andalucía los médicos rurales se ven promocionados mucho antes que los urbanos (podrán pasar a un nivel superior con sólo tres años de consolidación del nivel, en lugar de los cinco exigidos al común); por ejemplo, en Baleares se aplica la carrera profesional a médicos fijos e interinos (con ciertas condiciones) de las fundaciones de la empresa pública Gestió Sanitaria de Mallorca. Con todo ello puede hablarse de 18 modelos de carrera profesional, que ahondan en la separación entre servicios de salud, y que dificultarán la libre circulación de médicos. Un observador ajeno podría llegar a la conclusión de que hay un decidido propósito en el logro de servicios sanitarios peculiares, por CCAA.

Por supuesto, las retribuciones anexas a las categorías son muy diferentes, siendo en Madrid donde se encuentran las más altas (respectivamente, en euros anuales, para las cuatro categorías, 4.100, 7.600, 10.700 y 13.500). En Extremadura encontramos las menores retribuciones: 2.640, 5.280, 8.040 y 10.560 euros anuales ${ }^{6}$. Las cantidades son muy distintas, y contribuyen a aumentar las diferencias retributivas entre médicos que teóricamente pertenecen al mismo Sistema Nacional de Salud. Todo ello sin causa que lo justifique, pues no se espera menos de un médico extremeño que de un médico madrileño, ni parece que en justicia puedan medirse los méritos de forma diferente, y resulte que los méritos de los médicos madrileños sean muchos mayores que los de los médicos extremeños. 


\section{LA FORMA DE PAGO, CLAVE PARA EL TRABAJO}

Son múltiples las formas de pago al médico, aunque las predominantes sean sólo tres: salario, capitación y pago por acto. Por supuesto, ninguna forma de pago es perfecta $^{16,17}$. Parece que lo mejor es una combinación de todas ellas, del estilo de lo que se hace para pagar a los médicos generales daneses, ingleses y holandeses: un pago por capitación fuerte, un complemento salarial, y distintos pagos por acto. La búsqueda de mejoras en la efectividad está llevando a una preocupación mundial acerca de nuevas formas de pago, bien por rendimiento (por calidad en la práctica clínica), bien por formas de organización (por desarrollo de áreas específicas de trabajo) ${ }^{18,19}$.

Se sobreentiende que tanto la forma de pago como las cantidades finales son determinantes en el trabajo diario, en lo que se puede exigir al médico. Todo ello bien lejos de la carrera profesional a la española. Es decir, bien lejos de la pura retribución dineraria no ajustada según nuevas tareas o mayor dedicación, o mejoras en la calidad. En este sentido, el incremento salarial de la carrera profesional puede verse como reparación de injusticias previas, pues no se acompaña de exigencia alguna en la práctica clínica diaria. Y es cierto que los médicos españoles somos los peor pagados de la Unión Europea desarrollada (UE-15), pero no parece prudente ir hacia la convergencia por puertas falsas.

Lo lógico es que la carrera profesional fuera tanto una forma de reconocimiento profesional como un estímulo para la competencia en el trabajo clínico. Por ello, debería ligarse el nivel a las capacidades, a las exigencias, a las expectativas de rendimiento. De esta forma, el cambio de categoría debería conllevar el cambio de responsabilidad y el cambio de organización. Por ejemplo, en los niveles altos el médico gozaría de la posibilidad de contar con una auxiliar de clínica en su consulta, y/o tendría derecho a manejar un presupuesto global que le permitiese elegir especialista para sus pacientes incluso en centros privados concertados, y/o se convertiría en miembro de los comités asesores del gerente, y/o se le concederían "privilegios" en las guardias, y/o se esperaría que ampliase su campo de trabajo $^{19}$. Lamentablemente, de ello no se habla cuando se trata de la carrera profesional. Todo ha quedado reducido a una compensación monetaria que no cambia la forma de trabajo en nada.

Al incrementar la remuneración sin modificar las tareas se produce una grave ineficiencia, y la sociedad emplea recursos sin exigir mejoras. Convendría distinguir entre la necesidad de ir equiparando la remuneración de los médicos españoles con los de la UE-15, y la exigencia ética y pragmática de ligar los aumentos al incremento de rendimiento.

\section{CONCLUSIÓN}

La carrera profesional ha sido una oportunidad perdida, pues no va ligada a cambios en el trabajo, ha incre- mentado las diferencias autonómicas, ha hecho más iguales a los desiguales, ha discriminado a los interinos, y no tendrá impacto en la salud de la población. Además, los criterios empleados en la carrera profesional apenas tienen en cuenta el trabajo del médico de a pie, del médico clínico que encuentra satisfacción en el trabajo diario bien hecho. Es hora de empezar a introducir cambios que reparen estos errores.

\section{BIBLIOGRAFÍA}

1. Simó J, Campos JC, Sanfélix J. Carrera profesional y médicos de familia: reflexiones y propuestas. (I). Aten Primaria. 2002; 29:105-15.

2. Simó J, Campos JC, Sanfélix J. Carrera profesional y médicos de familia: reflexiones y propuestas. (II): Aten Primaria. 2002; 29:172-7.

3. Simó J, Casado V. Carreras profesionales: algo más que reconocimiento. Aten Primaria. 2006; 38:405-8.

4. González B, Barber P. Desigualdades territoriales en el Sistema Nacional de Salud de España. Madrid: Fundación Alternativas, Documento de Trabajo 90; 2006.

5. Martín Martín JJ. Autonomía y desigualdades en salud. Gestión Clín Sanit. 2007; 9:127-31.

6. San Segundo G. 18 formas de tratar a los médicos. Med Economics (Ed Esp). 2007; 4(19):22-9.

7. Ortún V. (Nuevas) formas de investigación en atención primaria. Segundo Seminario de Innovación en Atención Primaria 2007. En: www.fcs.

8. Gérvas J, Ortún V, Palomo L, Ripoll MA, Seminarios Innovación Atención Primaria 2007. Incentivos en atención primaria: de la contención del gasto a la salud de la población. Rev Esp Salud Pública. 2007; 81:589-96.

9. Sen A. Development of freedom. Oxford: OUP; 1999.

10. Durán A, Gérvas J. Acerca de la transferencia de experiencias del oeste al este europeo. Algunos errores evitables al asesorar sobre reformas sanitarias. Gac Sanit. 2006; 20:503-9.

11. Franco A, Álvarez-Dardet C, Ruiz MT. Effect of democracy on health: an ecological study. BMJ. 2004; 329:1241-4.

12. Gérvas J, Ortún V. Propuesta de incentivos para una Medicina General por cuenta ajena. Gac Sanit. 1996; 10:349.

13. FADSP. Informe: Evaluación de la Atención Primaria en las CCCAA. Madrid: FADSP; 2007. En: www.fadsp.org/pdf/ APCCAA07.doc

14. Martín M, Sánchez-Bayle M, Palomo L. El desarrollo de la Atención Primaria en relación a la orientación política de los Gobiernos Autonómicos. Aten Primaria. 2008 [en prensa].

15. Gérvas J. Salud y política, una relación sin inocencia. Aten Primaria. 2008; 40:277-84.

16. Pellisé L. Sistemas de pago óptimos en el sector sanitario. En: Llano L, Ortún V, Martín JM, Millán J, Gené J (eds). Barcelona: Masson; 1998. págs. 49-63.

17. Palomo L. Pagar a los médicos. Salud 2000. 2000; 78:610.

18. Fleetcroft $R$, Cookson $R$. Do the incentive payments in the new NHS contract for primary care reflect likely population health gain? J Health Serv Res. 2006; 11:27-31.

19. Gérvas J, Starfield B, Violán C, Minué S. GPs with special interests: unanswered questions. Br J Gen Pract. 2007; 57:912-7.

20. Gérvas J, Simó J. 2015, el día a día de un médico de cabecera. SEMERGEN. 2005; 31:478-85. 